\title{
Distributionally regulated functions
}

\author{
by \\ Jasson Vindas and Ricardo Estrada (Baton Rouge, LA)
}

\begin{abstract}
We study the class of distributions in one variable that have distributional lateral limits at every point, but which have no Dirac delta functions or derivatives at any point, the "distributionally regulated functions." We also consider the related class where Dirac delta functions are allowed. We prove several results on the boundary behavior of functions of two variables $F(x, y), x \in \mathbb{R}, y>0$, with $F\left(x, 0^{+}\right)=f(x)$ distributionally, both near points where the distributional point value exists and points where the lateral distributional limits exist. We give very general formulas for the jumps, in terms of $F$ and related functions. We prove that the set of singular points of a distributionally regulated function is always countable at the most. We also characterize the Fourier transforms of tempered distributionally regulated functions in two ways.
\end{abstract}

1. Introduction. The theory of distributions is one of the most powerful tools available in applied mathematics. Ever since their introduction, distributions have shown their usefulness; actually, this was true even before their formal introduction [23]. Many textbooks cover the theory and applications of distributions [5, 14, 19, 20, 30, 38].

There are several approaches to the theory of distributions, but in all of them one quickly learns that distributions do not have point values, as functions do, despite the fact that they are sometimes called generalized "functions." Interestingly, many common objects in analysis do not have point values, even though they are referred as "functions": If $f \in L^{1}(\mathbb{R})$, what is $f(0)$ ? Recall that the elements of $L^{1}(\mathbb{R})$ are equivalence classes of functions equal almost everywhere, and thus one may change the values on any set of measure zero, like $\{0\}$ for instance, without changing the element of $L^{1}(\mathbb{R})$.

In a seminal work, Łojasiewicz [21] was the first to give a satisfactory definition of the value of a distribution at a point, which when applied at points where the distribution is locally equal to a continuous function gives the usual value, but can also be applied in more complicated situations.

2000 Mathematics Subject Classification: Primary 46F10.

Key words and phrases: distributions, point values, regulated functions. 
The notion of point value in the sense of Łojasiewicz has been shown to be very useful in several areas, such as abelian and tauberian results for integral transforms [24, 27, 31, 36], spectral expansions [14, 32], the boundary behavior of solutions of partial differential equations [11,33], or the summability of cardinal series $[34,35]$. It is remarkable that there is a characterization of the Fourier series of distributions having a value at a point [8], while no corresponding results are known for other notions of value. The notion of distributional point value of Łojasiewicz has been generalized in several directions, like the idea of distributionally bounded distributions [5], and especially the theory of distributional asymptotic expansions developed by several authors $[14,28,31]$.

In [21], Łojasiewicz also introduced and studied the class of distributions that have a value at every point. As he showed, these distributions deserve to be called "functions" since the function given by its values is a well-defined measurable function, and the correspondence between the distributions with values at every point and the function of its values is a bijection. Although there is a notion, that of regular distribution, that appears to apply exactly to those distributions that correspond to functions, it is fair to say that the distributions introduced by Łojasiewicz, even if not "regular," are objects that one would call "functions."

The aim of this article is to introduce and study a generalization of the Łojasiewicz functions, namely the distributionally regulated functions, which are those distributions that have a distributional lateral limit at every point without having Dirac delta functions or derivatives at any point. We also consider the related class of distributionally regulated functions with delta functions, which are those distributions that have a distributional lateral limit at every point; we show that in this case the set of points where there are delta functions is countable at the most. If $f$ is a distributionally regulated function (without delta functions), with lateral limits $f\left(x^{+}\right)$and $f\left(x^{-}\right)$at each $x \in \mathbb{R}$, then we introduce the function

$$
\widetilde{f}(x)=\frac{f\left(x^{+}\right)+f\left(x^{-}\right)}{2} .
$$

The function $\widetilde{f}$ is a well-defined measurable function, and the correspondence $f \leftrightarrow \widetilde{f}$ is one-to-one and onto. Therefore, it is justified to identify the distribution $f$ and the function $\widetilde{f}$, and call $f$ a "function." When $f$ is a distributionally regulated function with delta functions, then $\widetilde{f}$ captures the ordinary function part of $f$, and $f-\widetilde{f}$ is a singular distribution that consists of sums of Dirac delta functions and derivatives on some at most countable set. The distributionally regulated functions also generalize the classical regulated functions, which are those functions that have ordinary lateral limits at every point [6]. The classical regulated functions play a role in many areas 
of mathematics such as conformal mapping theory [29], in the description of curves by their radius of curvature [12] and the application of these ideas to the study of crystals [37], and in theories of integration more general than the Lebesgue integral, a subject that has received increased attention in recent years $[1,17]$. Actually, Łojasiewicz proved that there is a descriptive integral that can be defined for distributions that have a value at every point, and it is easy to see that this integral is also defined for distributionally regulated functions. For this integral one has

$$
\langle f(x), \phi(x)\rangle=\int_{-\infty}^{\infty} \widetilde{f}(x) \phi(x) d x
$$

for any test function $\phi \in \mathcal{D}(\mathbb{R})$.

The article is organized as follows. In Section 2 we give some preliminary notions on distributions, point values and the Cesàro behavior of distributions. Distributionally regulated functions are defined in Section 3. The next section introduces the $\phi$-transform, a function of two variables $F(x, y)$, $x \in \mathbb{R}, y>0$, that satisfies $F\left(x, 0^{+}\right)=f(x)$ distributionally and that allows us to study the local behavior of a distribution $f$. In Sections 4 and 5 we consider the pointwise boundary behavior of $F(x, y)$ as $(x, y)$ approaches the point $\left(x_{0}, 0\right)$ in the cases when the distributional value $f\left(x_{0}\right)$ exists and when just the distributional limits $f\left(x_{0}^{ \pm}\right)$exist. We give several formulas for the distributional jumps of $f$ in terms of the $\phi$-transform and related functions; these formulas are very general versions of the jump formulas initially given by Fejér [15], [39, 9.11] and by Lukács [22], [39, Thm. 8.13] for the ordinary jumps of a Fourier series, and recently generalized by Móricz [25], [11]. Our formulas apply to distributions with arbitrary support, which do not need to be periodic, and are given not only in terms of conjugate harmonic functions but in terms of more general solutions of partial differential equations, as follows from the results of Section 7 .

In Section 6 we show that the set of singular points of a distributionally regulated function, namely where the lateral limits do not coincide, or where there are delta functions, is countable at the most; this result is easily proved for classical regulated functions, but a new proof is required in this case. In Section 7 we show that the $\phi$-transform is often a solution of a partial differential equation, such as the Laplace equation or the heat equation, and therefore our results become results on the boundary behavior of solutions of partial differential equations. Finally, in Section 8 we provide two characterizations of the Fourier transform of tempered distributionally regulated functions. One is in terms of the existence of the limits of integrals of the type $\lim _{s \rightarrow \infty} \int_{-s}^{a s} \widehat{f}(u) e^{-i u x} d u$, in the Cesàro sense, for $a>0$, the other in terms of the decomposition of the distribution $\widehat{f}(u)$ in terms of boundary values of analytic functions from the upper and lower half planes. 
2. Preliminaries. In this section we describe the spaces of test functions and distributions needed in this paper. We also give a summary of the notion of Cesàro behavior of a distribution at infinity [9] and at a point [14, 21]. All of our functions and distributions are over one-dimensional spaces.

The spaces of test functions $\mathcal{D}, \mathcal{E}$, and $\mathcal{S}$ and the corresponding spaces of distributions $\mathcal{D}^{\prime}, \mathcal{E}^{\prime}$, and $\mathcal{S}^{\prime}$ are well-known [19, 20, 30]. In general [38] we call a topological vector space $\mathcal{A}$ a space of test functions if $\mathcal{D} \subset \mathcal{A} \subset \mathcal{E}$, the inclusions being continuous, and if the derivative $d / d x$ is a continuous operator of $\mathcal{A}$. Another useful space, particularly in the study of distributional asymptotic expansions $[14,28,31]$, is $\mathcal{K}^{\prime}$, the dual of $\mathcal{K}$. A smooth function $\phi$ belongs to $\mathcal{K}$ if there is a constant $\gamma$ such that $\phi^{(k)}(x)=O\left(|x|^{\gamma-k}\right)$ as $|x| \rightarrow \infty$ for $k=0,1,2, \ldots$, that is, if $\phi(x)=O\left(|x|^{\gamma}\right)$ strongly. The space $\mathcal{K}$ is formed by the so-called GLS symbols [18]; the topology of $\mathcal{K}$ is given by the canonical seminorms. The space $\mathcal{K}^{\prime}$ plays a fundamental role in the theory of summability of distributional evaluations [9]. The elements of $\mathcal{K}^{\prime}$ are exactly the generalized functions that decay very rapidly at infinity in the distributional sense or, equivalently, in the Cesàro sense.

The Cesàro behavior of a distribution at infinity is studied by using the order symbols $O\left(x^{\alpha}\right)$ and $o\left(x^{\alpha}\right)$ in the Cesàro sense. If $f \in \mathcal{D}^{\prime}(\mathbb{R})$ and $\alpha \in \mathbb{R} \backslash\{-1,-2,-3, \ldots\}$, we say that $f(x)=O\left(x^{\alpha}\right)$ as $x \rightarrow \infty$ in the Cesàro sense and write

$$
f(x)=O\left(x^{\alpha}\right) \quad(\mathrm{C}) \text { as } x \rightarrow \infty
$$

if there exists $N \in \mathbb{N}$ such that every primitive $F$ of order $N$ of $f$, i.e., $F^{(N)}=f$, is an ordinary function for large arguments and satisfies the ordinary order relation

$$
F(x)=p(x)+O\left(x^{\alpha+N}\right) \quad \text { as } x \rightarrow \infty
$$

for a suitable polynomial $p$ of degree $N-1$ at the most. When the value of $N$ is important we use the notation

$$
f(x)=O\left(x^{\alpha}\right) \quad(\mathrm{C}, N) \text { as } x \rightarrow \infty .
$$

A similar definition applies to the little $o$ symbol. The definitions when $x \rightarrow-\infty$ are clear. One can also consider the case when $\alpha=-1,-2,-3, \ldots$ [14, Def. 6.3.1].

The equivalent notations $f(x)=O\left(x^{-\infty}\right)$ and $f(x)=o\left(x^{-\infty}\right)$ mean that $f(x)=O\left(x^{-\beta}\right)$ for each $\beta>0$. It is shown in [9], [14, Thm. 6.7.1] that a distribution $f \in \mathcal{D}^{\prime}$ is of rapid decay at $\pm \infty$ in the (C) sense,

$$
f(x)=O\left(|x|^{-\infty}\right) \quad(\mathrm{C}) \text { as }|x| \rightarrow \infty,
$$

if and only if $f \in \mathcal{K}^{\prime}$. Functions like $\sin x, J_{0}(x)$, or $x^{2} e^{i x}$ belong to $\mathcal{K}^{\prime}$ and thus are "distributionally small." The space $\mathcal{K}^{\prime}$ is a distributional analogue of the space $\mathcal{S}$ of rapidly decreasing smooth functions [14, Section 2.9]. 
These ideas can be readily extended to the study of the local behavior of generalized functions [14, 31]. Actually, Łojasiewicz [21] defined the value of a distribution $f \in \mathcal{D}^{\prime}(\mathbb{R})$ at a point $x_{0}$ as the limit

$$
f\left(x_{0}\right)=\lim _{\varepsilon \rightarrow 0} f\left(x_{0}+\varepsilon x\right),
$$

if the limit exists in $\mathcal{D}^{\prime}(\mathbb{R})$, that is, if

$$
\lim _{\varepsilon \rightarrow 0}\left\langle f\left(x_{0}+\varepsilon x\right), \phi(x)\right\rangle=f\left(x_{0}\right) \int_{-\infty}^{\infty} \phi(x) d x
$$

for each $\phi \in \mathcal{D}(\mathbb{R})$. He showed that the existence of the distributional point value $\gamma=f\left(x_{0}\right)$ is equivalent to the existence of $n \in \mathbb{N}$, and a primitive of order $n$ of $f$, that is, $F^{(n)}=f$, which is continuous near $x=x_{0}$ and satisfies

$$
\lim _{x \rightarrow x_{0}} \frac{n ! F(x)}{\left(x-x_{0}\right)^{n}}=\gamma
$$

For example the generalized function $f(x)=\sin (1 / x)$ is oscillatory near $x=0$, however, it is easy to see that $f(0)$ exists and equals 0 .

More generally, one could try to look for a representation of the form

$$
f\left(x_{0}+\varepsilon x\right) \sim \varepsilon^{\delta} g(x) \quad \text { as } \varepsilon \rightarrow 0
$$

in the space $\mathcal{D}^{\prime}(\mathbb{R})$, where $g$ is non-null. One can then show that $g$ has to be homogeneous of order $\delta$. When $f\left(x_{0}+\varepsilon x\right)=o\left(\varepsilon^{\delta}\right)$ as $\varepsilon \rightarrow 0^{+}$, because of equivalences similar to (2.7), we sometimes write $f\left(x_{0}+x\right)=o\left(x^{\beta}\right)(\mathrm{C})$ as $x \rightarrow 0^{+}[14$, Thm. 6.9.1].

If we consider the limit of $f\left(x_{0}+\varepsilon x\right)$ in $\mathcal{D}^{\prime}(\mathbb{R} \backslash\{0\})$, then we obtain the concept of the distributional limit of $f(x)$ at $x=x_{0}$. Thus $\lim _{x \rightarrow x_{0}} f(x)=L$ distributionally if

$$
\lim _{\varepsilon \rightarrow 0}\left\langle f\left(x_{0}+\varepsilon x\right), \phi(x)\right\rangle=L \int_{-\infty}^{\infty} \phi(x) d x, \quad \phi \in \mathcal{D}(\mathbb{R} \backslash\{0\}) .
$$

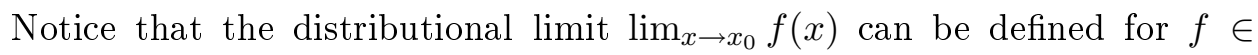
$\mathcal{D}^{\prime}\left(\mathbb{R} \backslash\left\{x_{0}\right\}\right)$. If the point value $f\left(x_{0}\right)$ exists distributionally then the distri-

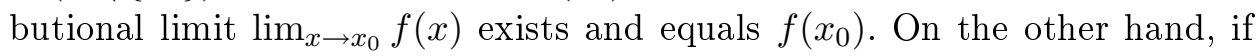
$\lim _{x \rightarrow x_{0}} f(x)=L$ distributionally then there exist constants $a_{0}, \ldots, a_{n}$ such that $f(x)=f_{0}(x)+\sum_{j=0}^{n} a_{j} \delta^{(j)}\left(x-x_{0}\right)$, where the distributional point value $f_{0}\left(x_{0}\right)$ exists and equals $L$.

We may also consider lateral limits. We say that the distributional lateral value $f\left(x_{0}^{+}\right)$exists if $f\left(x_{0}^{+}\right)=\lim _{\varepsilon \rightarrow 0^{+}} f\left(x_{0}+\varepsilon x\right)$ in $\mathcal{D}^{\prime}(0, \infty)$, that is,

$$
\lim _{\varepsilon \rightarrow 0^{+}}\left\langle f\left(x_{0}+\varepsilon x\right), \phi(x)\right\rangle=f\left(x_{0}^{+}\right) \int_{0}^{\infty} \phi(x) d x, \quad \phi \in \mathcal{D}(0, \infty) .
$$


Similar definitions apply to $f\left(x_{0}^{-}\right)$. Notice that the distributional limit $\lim _{x \rightarrow x_{0}} f(x)$ exists if and only if the distributional lateral limits $f\left(x_{0}^{-}\right)$and $f\left(x_{0}^{+}\right)$exist and coincide.

3. Regulated functions. In his pioneering work, Łojasiewicz [21] introduced and studied the distributions that have a distributional point value at every point. He proved that if one considers the function having those distributional values as values, then this function is measurable and in a very precise sense, the distribution corresponds to the function. It is common usage to call a distribution "regular" if it arises from a locally Lebesgue integrable function. The functions studied by Łojasiewicz are more general instances of what one should call "regular" distributions, namely those arising from a function by integration. However, in general, the functions that arise from the distributional point values are often not locally integrable in the sense of Lebesgue; sometimes they are locally integrable with respect to more general integration processes such as the Denjoy-Perron-Henstock integral, like the function $f_{1}(x)=x^{-1} \sin x^{-1}, x \neq 0, f_{1}(0)=0$, but sometimes they are not, like $f_{2}(x)=x^{-2} \sin x^{-1}, x \neq 0, f_{2}(0)=0$.

In this article we shall study a somewhat bigger class, that of distributionally regulated functions. The definition is as follows.

Definition. A distribution $f \in \mathcal{D}^{\prime}(\mathbb{R})$ is called a distributionally regulated function if at each point $x_{0} \in \mathbb{R}$ both distributional lateral limits $f\left(x_{0}^{ \pm}\right)$exist and $f$ has no Dirac delta functions at $x=x_{0}$. We say that $f$ is a distributionally regulated function with delta functions if at each point $x_{0} \in \mathbb{R}$ both distributional lateral limits $f\left(x_{0}^{ \pm}\right)$exist.

It will follow from our study that a distribution that is a distributionally regulated function actually corresponds to an actual function, the function given by the distributional point value $f\left(x_{0}\right)$, which is defined whenever $f\left(x_{0}^{+}\right)=f\left(x_{0}^{-}\right)$, an equation that holds for all $x_{0}$ except for those of an exceptional set that is countable at the most.

On the other hand, a distributionally regulated function with delta functions is a distribution, and the name "function" is used in the way the name function is used for the Dirac delta function.

Sometimes we shall refer to distributionally regulated functions as "distributionally regulated functions without delta functions."

The distributionally regulated functions that have no distributional jump at any point are the functions studied in [21], and therefore we shall call them Eojasiewicz functions.

Our definitions were given for a distribution $f \in \mathcal{D}^{\prime}(\mathbb{R})$, defined over the whole real line. However, one can consider any of these notions over finite intervals in the obvious way, namely, a distribution is, say, a distributionally 
regulated function over the interval $(a, b)$ if its distributional lateral limits exist at each point, and no delta functions are present.

It is worth pointing out that the classical regulated functions are those classical functions that have lateral limits at every point. They are precisely the uniform limits of step functions [6]. Observe that the classical analogue of the Lojasiewicz functions are the continuous functions.

EXAmple. If $a, b, c, d$ are constants, and $H$ is the Heaviside function, then

$$
f_{0}(x)=\left(a+b \sin \frac{1}{x}\right) H(x)+\left(c+d \sin \frac{1}{x}\right) H(-x)
$$

is a distributionally regulated function; it is not a classical regulated function and it is not a function of bounded variation. One can use some condensation of singularities technique to obtain examples that show this behavior not only at $x=0$ but over a dense set. For instance, if $\left\{\omega_{n}\right\}_{n=0}^{\infty}$ is dense in $\mathbb{R}$, and if $\sum_{n=0}^{\infty}\left|a_{n}\right|<\infty$, then

$$
f_{1}(x)=\sum_{n=0}^{\infty} a_{n} f_{0}\left(x-\omega_{n}\right)
$$

is a distributionally regulated function with distributional jumps at the points $x=\omega_{n}$. Similarly, if $q>1$, the function

$$
f_{2}(x)=\sum_{n=1}^{\infty} \frac{f_{0}(\sin n x)}{n^{q}}
$$

is continuous at all the irrational points and has distributional jump discontinuities at each rational number.

4. The $\phi$-transform. Our main tool to study the local behavior of distributions is the $\phi$-transform, a function of two variables that we now define.

Let $\phi \in \mathcal{D}(\mathbb{R})$ be a fixed test function that satisfies

$$
\int_{-\infty}^{\infty} \phi(x) d x=1
$$

If $f \in \mathcal{D}^{\prime}(\mathbb{R})$ we introduce the function of two variables $F=F_{\phi}\{f\}$ by the formula

$$
F(x, y)=\langle f(x+y \xi), \phi(\xi)\rangle, \quad x \in \mathbb{R}, y>0,
$$

the distributional evaluation with respect to the variable $\xi$. We call $F$ the $\phi$-transform of $f$.

The $\phi$-transform can also be defined if $\phi$ does not belong to $\mathcal{D}(\mathbb{R})$ as long as we consider only distributions $f$ of a more restricted class. Indeed, we can 
consider the case when $\phi \in \mathcal{A}(\mathbb{R})$ and $f \in \mathcal{A}^{\prime}(\mathbb{R})$ for any suitable space $\mathcal{A}(\mathbb{R})$ of test functions, such as $\mathcal{S}(\mathbb{R}), \mathcal{K}(\mathbb{R})$, or $\mathcal{E}(\mathbb{R})$. Observe that we assume (4.1) in every case.

Our first result shows that $f(x)$ is the distributional boundary value of $F(x, y)$ as $y \rightarrow 0$. then

Theorem 1. If $f \in \mathcal{D}^{\prime}(\mathbb{R})$ and $F$ is its $\phi$-transform defined by (4.2)

$$
\lim _{y \rightarrow 0} F(x, y)=f(x)
$$

distributionally in the space $\mathcal{D}^{\prime}(\mathbb{R})$, that is,

$$
\lim _{y \rightarrow 0}\langle F(x, y), \psi(x)\rangle=\langle f(x), \psi(x)\rangle, \quad \forall \psi \in \mathcal{D}(\mathbb{R}) .
$$

Proof. If $\psi \in \mathcal{D}(\mathbb{R})$ then

$$
\langle F(x, y), \psi(x)\rangle=\langle\Psi(y \xi), \phi(\xi)\rangle,
$$

where

$$
\Psi(z)=\langle f(x), \psi(x-z)\rangle
$$

is a smooth function of $z$. Therefore, $\Psi(0)$ exists in the ordinary sense and consequently in the distributional sense of Łojasiewicz. Hence,

$$
\lim _{y \rightarrow 0}\langle\Psi(y \xi), \phi(\xi)\rangle=\Psi(0)=\langle f(x), \psi(x)\rangle,
$$

and (4.4) follows.

The result will also hold when $f \in \mathcal{E}^{\prime}(\mathbb{R})$ and $\phi \in \mathcal{E}(\mathbb{R})$ if $\phi \in L^{1}(\mathbb{R})$. In that case (4.7) follows from the Lebesgue dominated convergence theorem, since $\Psi \in \mathcal{D}(\mathbb{R})$. Another case when $f(x)$ is the distributional boundary value of $F(x, y)$ as $y \rightarrow 0$ is if

$$
\begin{array}{ll}
f(x)=O\left(|x|^{\beta}\right) & (\mathrm{C}) \text { as }|x| \rightarrow \infty, \\
\phi(x)=O\left(|x|^{\alpha}\right) & \text { strongly as }|x| \rightarrow \infty,
\end{array}
$$

and

$$
\alpha<-1, \quad \alpha+\beta<-1,
$$

as follows from [11, Theorem 1]. It is true in particular if $f \in \mathcal{S}^{\prime}(\mathbb{R})$ and $\phi \in \mathcal{S}(\mathbb{R})$.

For future reference, we say that if $f \in \mathcal{D}^{\prime}(\mathbb{R})$ and $\phi \in \mathcal{D}(\mathbb{R})$ we are in Case I. If (4.8)-(4.10) are satisfied, we say that we are in Case II. When $f \in \mathcal{S}^{\prime}(\mathbb{R})$ and $\phi \in \mathcal{S}(\mathbb{R})$ we say that we are in Case III. Most of our results will hold in any of these three cases. However, the results are usually false when we just assume that $f \in \mathcal{E}^{\prime}(\mathbb{R})$ and $\phi \in \mathcal{E}(\mathbb{R})$.

Theorem 2. Suppose

$$
f\left(x_{0}\right)=\gamma
$$


distributionally. In any of Cases I, II, or III, we have

$$
\lim _{(x, y) \rightarrow\left(x_{0}, 0\right)} F(x, y)=\gamma
$$

in any sector $y \geq m\left|x-x_{0}\right|$ for any $m>0$.

Proof. Let us show that if $\left|x_{1}\right| \leq 1 / m$ then $\lim _{\varepsilon \rightarrow 0^{+}} F\left(x_{0}+\varepsilon x_{1}, \varepsilon\right)=\gamma$. Indeed, if $\phi \in \mathcal{D}(\mathbb{R})$, then

$$
\begin{aligned}
F\left(x_{0}+\varepsilon x_{1}, \varepsilon\right) & =\left\langle f\left(x_{0}+\varepsilon x_{1}+\varepsilon \xi\right), \phi(\xi)\right\rangle=\left\langle f\left(x_{0}+\varepsilon \omega\right), \phi\left(\omega-x_{1}\right)\right\rangle \\
& =\left\langle f\left(x_{0}+\varepsilon \omega\right), \phi_{x_{1}}(\omega)\right\rangle
\end{aligned}
$$

where $\phi_{x_{1}}(\omega)=\phi\left(\omega-x_{1}\right)$ also belongs to $\mathcal{D}(\mathbb{R})$ and $\int_{-\infty}^{\infty} \phi_{x_{1}}(\omega) d \omega=1$. Thus (4.12) follows. The limit is uniform with respect to $x_{1}$ for $\left|x_{1}\right| \leq 1 / m$ since $\left\{\phi_{x_{1}}:\left|x_{1}\right| \leq 1 / m\right\}$ is a compact set in $\mathcal{D}(\mathbb{R})$. The proof in Cases II and III is similar.

Angular convergence of $F(x, y)$ to $\gamma=f\left(x_{0}\right)$ is obtained when the distributional point value exists. On the other hand, the radial limit, $\lim _{y \rightarrow 0^{+}} F\left(x_{0}, y\right)$, exists under a weaker hypothesis.

TheOREM 3. Suppose Case I, II, or III holds, and the test function $\phi$ is even. Let $\chi_{x_{0}}(s)=\left(f\left(x_{0}+s\right)+f\left(x_{0}-s\right)\right) / 2$. If

$$
\chi_{x_{0}}(0)=\gamma
$$

distributionally, then

$$
\lim _{y \rightarrow 0^{+}} F\left(x_{0}, y\right)=\gamma .
$$

Proof. The fact that $\phi$ is even yields

$$
\begin{aligned}
\lim _{y \rightarrow 0^{+}} F\left(x_{0}, y\right) & =\lim _{y \rightarrow 0^{+}}\left\langle f\left(x_{0}+y \xi\right), \phi(\xi)\right\rangle \\
& =\lim _{y \rightarrow 0^{+}}\left\langle f\left(x_{0}+y \xi\right),(\phi(\xi)+\phi(-\xi)) / 2\right\rangle \\
& =\lim _{y \rightarrow 0^{+}}\left\langle\chi_{x_{0}}(y \xi), \phi(\xi)\right\rangle=\gamma,
\end{aligned}
$$

as required.

REMARK. The above result does not hold if $f \in \mathcal{E}^{\prime}(\mathbb{R})$ and $\phi \in \mathcal{E}(\mathbb{R})$. Indeed, if

$$
\phi(x)=\frac{3 \sin x^{3}}{\pi x},
$$

then $\phi \in \mathcal{E}$ and $\int_{-\infty}^{\infty} \phi(x) d x=1$. If $f(x)=\delta(x)$, then

$$
F(x, y)=\left(\frac{3}{\pi x}\right) \sin \left(\frac{x}{y}\right)^{3} .
$$

If $x_{0} \neq 0$ then $f\left(x_{0}\right)=0$ but even the radial $\operatorname{limit}_{\lim _{y \rightarrow 0^{+}}} F\left(x_{0}, y\right)$ does not exist. 
Suppose now that the distribution $f \in \mathcal{D}^{\prime}(\mathbb{R})$ has lateral distributional limits $f\left(x_{0}^{ \pm}\right)=\gamma_{ \pm}$as $x \rightarrow x_{0}$ from the right and from the left, respectively, and no delta functions at $x=x_{0}$. This means that for each $\psi \in \mathcal{D}(\mathbb{R})$,

$$
\lim _{\varepsilon \rightarrow 0^{+}}\left\langle f\left(x_{0}+\varepsilon \xi\right), \psi(\xi)\right\rangle=\gamma_{-} \int_{-\infty}^{0} \psi(\xi) d \xi+\gamma_{+} \int_{0}^{\infty} \psi(\xi) d \xi .
$$

Then we have the ensuing result.

THEOREM 4. Suppose Case I, II, or III holds and $f$ satisfies (4.17). Then for each $\theta \in(0, \pi)$ there exists $\alpha=\alpha(\theta) \in[0,1]$ such that

$$
\lim _{\substack{(x, y) \rightarrow\left(x_{0}, 0\right) \\(x, y) \in I_{\theta}}} F(x, y)=\alpha(\theta) \gamma_{+}+(1-\alpha(\theta)) \gamma_{-}
$$

where $\mathrm{I}_{\theta}$ is the line $y=\tan \theta\left(x-x_{0}\right)$.

In Cases II or III, $\lim _{\theta \rightarrow 0} \alpha(\theta)=1, \lim _{\theta \rightarrow \pi} \alpha(\theta)=0$. In Case I actually there exist $\theta_{0}, \theta_{1} \in(0, \pi)$ such that $\alpha(\theta)=1$ for $\theta \leq \theta_{0}$ while $\alpha(\theta)=0$ for $\theta \geq \theta_{1}$.

If $\phi$ is even then $\alpha(\pi / 2)=1 / 2$.

Proof. The limit of $F(x, y)$ as $(x, y) \rightarrow\left(x_{0}, 0\right)$ along $\mathrm{I}_{\theta}$ is given as

$$
\begin{aligned}
\lim _{\varepsilon \rightarrow 0^{+}}\left\langle f\left(x_{0}+\varepsilon \cos \theta+\varepsilon \sin \theta \xi\right), \phi(\xi)\right\rangle & =\lim _{\varepsilon \rightarrow 0^{+}}\left\langle f\left(x_{0}+\varepsilon \omega\right), \phi_{\theta}(\omega)\right\rangle \\
& =\gamma_{-} \int_{-\infty}^{0} \phi_{\theta}(\omega) d \omega+\gamma_{+} \int_{0}^{\infty} \phi_{\theta}(\xi) d \omega,
\end{aligned}
$$

where

$$
\phi_{\theta}(\omega)=\frac{1}{\sin \theta} \phi\left(\frac{\omega-\cos \theta}{\sin \theta}\right) .
$$

The result follows by taking

$$
\alpha(\theta)=\int_{0}^{\infty} \phi_{\theta}(\omega) d \omega=\int_{-\cot \theta}^{\infty} \phi(\omega) d \omega
$$

which has the stated properties.

REMARK. If $f\left(x_{0}^{ \pm}\right)=\gamma_{ \pm}$exist distributionally, then $f(x)=f_{0}(x)+$ $\sum_{j=0}^{m} c_{j} \delta^{(j)}\left(x-x_{0}\right)$ where $f_{0}$ has no delta functions at $x=x_{0}$. It follows that

$$
F(x, y)=F_{0}(x, y)+\sum_{j=0}^{m} \frac{c_{j}}{y^{j+1}} \phi^{(j)}\left(\frac{x_{0}-x}{y}\right) .
$$

Therefore (4.18) is still valid for the finite part of the limit:

$$
\text { F.p. } \lim _{\substack{\left.(x, y) \rightarrow\left(x_{0}, 0\right) \\(x, y) \in\right|_{\theta}}} F(x, y)=\alpha(\theta) \gamma_{+}+(1-\alpha(\theta)) \gamma_{-} \text {. }
$$


REMARK. If $\phi$ is even and $f\left(x_{0}^{ \pm}\right)=\gamma_{ \pm}$exist distributionally while $f$ has no delta functions at $x=x_{0}$ then (4.18) shows that the radial limit $\lim _{y \rightarrow 0^{+}} F\left(x_{0}, y\right)$ exists and equals $\left(\gamma_{+}+\gamma_{-}\right) / 2$. However, Theorem 3 is a stronger result, since the lateral limits may not exist if $\chi_{x_{0}}(s)$ has the distributional limit $\gamma$ at $s=0$. More generally, if

$$
\lim _{s \rightarrow 0^{+}} \chi_{x_{0}}(s)=\gamma
$$

distributionally, then

$$
\text { F.p. } \lim _{y \rightarrow 0^{+}} F\left(x_{0}, y\right)=\gamma \text {. }
$$

REMARK. If $f$ is a distributionally regulated function with delta functions then the finite part limit F.p. $\lim _{y \rightarrow 0^{+}} F(x, y)$ exists for each $x \in \mathbb{R}$, and equals $\left(f\left(x_{0}^{+}\right)+f\left(x_{0}^{-}\right)\right) / 2$. It will follow from the results of Section 6 that the set of points where the limit is not an ordinary limit is countable at the most. If $f$ is a distributionally regulated function without delta functions then the limit is an ordinary limit for each $x \in \mathbb{R}$. On the other hand, if $f$ is a distributionally regulated function without delta functions then $\lim _{(x, y) \rightarrow\left(x_{0}, 0\right),(x, y) \in \mathrm{I}} F(x, y)$ exists for each non-horizontal line I, and the set of points where the limit is not independent of $I$ is countable at the most, while if $f$ is a Eojasiewicz function then the limit is independent of I for each $x_{0} \in \mathbb{R}$.

5. Limits and jumps. Suppose $f \in \mathcal{D}^{\prime}(\mathbb{R})$ is such that the lateral limits $f\left(x_{0}^{ \pm}\right)=\gamma_{ \pm}$exist distributionally. In this section we consider certain formulas for the jump $d=[f]_{x=x_{0}}=\gamma_{+}-\gamma_{-}$in terms of the radial limits of some functions related to $F(x, y)$.

Let us start with the case when $f$ does not have delta functions at $x=x_{0}$. Observe that sometimes we shall use the notation $F_{, x}$ or $F_{, y}$ for the partial derivatives $\partial F / \partial x$ and $\partial F / \partial y$, respectively.

THEOREM 5. Let $f$ be a distribution and $\phi$ a test function that satisfies (4.1). Suppose Case I, II, or III holds. Suppose the distributional lateral limits $f\left(x_{0}^{ \pm}\right)=\gamma_{ \pm}$exist and $f$ has no delta functions at $x=x_{0}$. Let $d=\gamma_{+}-\gamma_{-}$be the jump of $f$ at $x=x_{0}$ and let $\nu=\phi(0)$. Then

$$
\lim _{y \rightarrow 0^{+}} y F_{, x}\left(x_{0}, y\right)=\nu d .
$$

Proof. The hypotheses yield the asymptotic formula

$$
f\left(x_{0}+\varepsilon x\right)=\gamma_{+} H(x)+\gamma_{-} H(-x)+o(1), \quad \varepsilon \rightarrow 0^{+},
$$

in the space $\mathcal{D}^{\prime}(\mathbb{R})$, where $H$ is the Heaviside function. Since distributional 
expansions can be differentiated, we obtain

$$
f^{\prime}\left(x_{0}+\varepsilon x\right)=\frac{d}{\varepsilon} \delta(x)+o\left(\frac{1}{\varepsilon}\right), \quad \varepsilon \rightarrow 0^{+} .
$$

Observe now that $F_{, x}$ is precisely the $\phi$-representation of $f^{\prime}(x)$. Thus $(5.3)$ yields

$$
F_{, x}\left(x_{0}, y\right)=\frac{d \phi(0)}{y}+o\left(\frac{1}{y}\right), \quad y \rightarrow 0^{+},
$$

and (5.1) follows.

If we just assume that the distributional lateral limits $f\left(x_{0}^{ \pm}\right)=\gamma_{ \pm}$exist, then $f$ may have delta functions at $x=x_{0}$ and thus the formula (5.1) can be modified by using the finite part of the limit:

$$
\text { F.p. } \lim _{y \rightarrow 0^{+}} y F_{, x}\left(x_{0}, y\right)=\nu d \text {. }
$$

Actually, to obtain (5.5) and in particular (5.1) there is no need to assume that the distributional lateral limits $f\left(x_{0}^{ \pm}\right)$exist; it is enough to suppose that the symmetric jump function

$$
\psi_{x_{0}}(s)=\frac{f\left(x_{0}+s\right)-f\left(x_{0}-s\right)}{2}
$$

has a distributional limit as $s \rightarrow 0$.

THEOREM 6. Let $f$ be a distribution and $\phi$ a test function that satisfies (4.1). Suppose Case I, II, or III holds. Suppose

$$
\psi_{x_{0}}\left(0^{+}\right)=d / 2
$$

distributionally. If $\phi$ is even then

$$
\text { F.p. } \lim _{y \rightarrow 0^{+}} y \frac{\partial F}{\partial x}\left(x_{0}, y\right)=\nu d .
$$

When $\psi_{x_{0}}(s)$ does not have delta functions at $s=0$ then (5.8) is an ordinary limit.

Proof. Indeed, the result follows by applying (5.5) or (5.1) to $\Psi(x, y)$, the $\phi$-representation of $\psi_{x_{0}}(x)$, and by observing that

$$
\begin{aligned}
F_{, x}\left(x_{0}, y\right) & =\left\langle f^{\prime}\left(x_{0}+y \xi\right), \phi(\xi)\right\rangle=\left\langle f^{\prime}\left(x_{0}+y \xi\right),(\phi(\xi)+\phi(-\xi)) / 2\right\rangle \\
& =\left\langle\left(f^{\prime}\left(x_{0}+y \xi\right)-f^{\prime}\left(x_{0}+y \xi\right)\right) / 2, \phi(\xi)\right\rangle=\left\langle\psi_{x_{0}}^{\prime}(y \xi), \phi(\xi)\right\rangle \\
& =\Psi_{, x}(0, y),
\end{aligned}
$$

since $\psi_{x_{0}}\left(0^{+}\right)=d / 2$.

Another formula for the jump is given in terms of logarithmic averages. Observe that in Case II, that is, $f(x)=O\left(|x|^{\beta}\right)(\mathrm{C})$ and $\phi(x)=$ $O\left(|x|^{\alpha}\right)$ strongly as $|x| \rightarrow \infty$, we need to assume not only that $\alpha<-1$ and $\alpha+\beta<-1$, but also that $\beta<0$. 
THEOREM 7. Let $f$ be a distribution and $\phi$ a test function that satisfies (4.1). Suppose Case I or Case II with $\beta<0$ holds. If $\psi_{x_{0}}\left(0^{+}\right)=d / 2$, then

$$
\text { F.p. } \lim _{y \rightarrow 0^{+}} \frac{1}{\ln y}\left\langle f\left(x_{0}+y \xi\right), \frac{\phi(\xi)-\phi(0)}{\xi}\right\rangle=\nu d .
$$

Proof. Observe that the condition $\beta<0$, or Case I, guarantees that the Cesàro evaluation $\left\langle f\left(x_{0}+y \xi\right), \varrho(\xi)\right\rangle$, where $\varrho(\xi)=(\phi(\xi)-\phi(0)) / \xi$, is well-defined. Notice also that if $f\left(x_{0}^{ \pm}\right)=\gamma_{ \pm}$exist and $f$ has no delta functions at $x=x_{0}$ then one may argue that $\left\langle f\left(x_{0}+y \xi\right), \varrho(\xi)\right\rangle$ approaches $\gamma_{-} \int_{-\infty}^{0} \varrho(\xi) d \xi+\gamma_{+} \int_{0}^{\infty} \varrho(\xi) d \xi$ as $y \rightarrow 0^{+}$; however, both integrals diverge: $\left|\int_{-\infty}^{0} \varrho(\xi) d \xi\right|=\left|\int_{0}^{\infty} \varrho(\xi) d \xi\right|=\infty$.

On the other hand,

$$
\begin{aligned}
\frac{\partial}{\partial y}\left\langle f\left(x_{0}+y \xi\right), \frac{\phi(\xi)-\phi(0)}{\xi}\right\rangle & =\left\langle\xi f^{\prime}\left(x_{0}+y \xi\right), \frac{\phi(\xi)-\phi(0)}{\xi}\right\rangle \\
& =\left\langle f^{\prime}\left(x_{0}+y \xi\right), \phi(\xi)-\phi(0)\right\rangle \\
& =\left\langle f^{\prime}\left(x_{0}+y \xi\right), \phi(\xi)\right\rangle=\frac{\partial F}{\partial x}\left(x_{0}, y\right) .
\end{aligned}
$$

Thus we may use the L'Hôpital rule to obtain

$$
\text { F.p. } \lim _{y \rightarrow 0^{+}} \frac{1}{\ln y}\left\langle f\left(x_{0}+y \xi\right), \frac{\phi(\xi)-\phi(0)}{\xi}\right\rangle=\text { F.p. } \lim _{y \rightarrow 0^{+}} y \frac{\partial F}{\partial x}\left(x_{0}, y\right)=\nu d,
$$

as required.

Remark. The function $\widetilde{F}(x, y)=\langle f(x+y \xi),(\phi(\xi)-\phi(0)) / \xi\rangle$ is a type of "conjugate" function to the $\phi$-transform $F(x, y)$. Actually if $\phi(x)=$ $\pi^{-1}\left(1+x^{2}\right)^{-1}$ then $F(x, y)$ is a harmonic function and $\widetilde{F}(x, y)$ is precisely its harmonic conjugate.

EXAMPLE. Let us consider the distributional behavior of the distribution $f_{\alpha}, \alpha>0$, given by the non-harmonic series

$$
f_{\alpha}(x)=\sum_{n=1}^{\infty} \frac{\sin n^{\alpha} x}{n}
$$

as $x \rightarrow 0$. Observe that $f_{\alpha}(x)=O\left(|x|^{-\infty}\right)(\mathrm{C})$ as $|x| \rightarrow \infty$. Let us consider the conjugate function $\widetilde{F}(x, y)$ with $\phi(x)=\pi^{-1}\left(1+x^{2}\right)^{-1}$ as in the remark above. Then

$$
\widetilde{F}(x, y)=\sum_{n=1}^{\infty} \frac{e^{-n^{\alpha} y} \cos n^{\alpha} x}{n}
$$

and thus $\widetilde{F}(0, y) \sim(1 / \alpha) \ln y$, since $\sum_{n^{\alpha} \leq N} 1 / n \sim(1 / \alpha) \ln N$ as $N \rightarrow \infty$, and it follows that $\nu d=1 / \alpha$, or $d=\pi / \alpha$, since $\phi(0)=1 / \pi$. Therefore, since 
$f_{\alpha}$ is odd, we obtain the distributional lateral limits

$$
f_{\alpha}\left(0^{+}\right)=\frac{\pi}{2 \alpha}, \quad f_{\alpha}\left(0^{-}\right)=\frac{-\pi}{2 \alpha} .
$$

Observe that this is easy to see for $\alpha=1$ from the well-known formula

$$
f_{1}(x)=\frac{\pi-x}{2}, \quad 0<x<\pi,
$$

and for $\alpha=1 / 2$ from the formula

$$
f_{1 / 2}(x)=\pi+\sum_{j=0}^{\infty} \frac{(-1)^{j} \zeta(1 / 2-j) x^{2 j+1}}{(2 j+1) !}, \quad x>0,
$$

obtained by Boersma [3] when solving a problem proposed by Glasser [16]; see also [7]. It is not hard to see that if $\alpha>1$ then (5.12) are not ordinary limits, since $f_{\alpha}$ is unbounded as $x \rightarrow 0$.

6. The number of singularities. In this section we show that if $f$ is a distributionally regulated function, with or without delta functions, then the distributional point value $f(x)$ exists for all $x$ apart from an exceptional set which is countable at the most.

The corresponding result for ordinary regulated functions is well-known, and actually very easy to prove. Indeed, if $f(x)$ is a regulated function in some interval $I$ then for any $\lambda>0$ the set $\mathfrak{S}_{\lambda}$ consisting of the points $x$ where $\left|f\left(x^{+}\right)-f\left(x^{-}\right)\right| \geq \lambda$ is discrete in $I$, since at an accumulation point of $\mathfrak{S}_{\lambda}$ at least one of the lateral limits cannot exist. Thus $\mathfrak{S}_{\lambda}$ is countable at the most, and hence so is $\mathfrak{S}=\bigcup_{\lambda>0} \mathfrak{S}_{\lambda}=\bigcup_{n=1}^{\infty} \mathfrak{S}_{1 / n}$. When $f$ is a regulated function of bounded variation, then one can even bound $n_{\lambda}(K)$, the number of elements of $\mathfrak{S}_{\lambda} \cap K$ for any compact interval $K$, by $n_{\lambda}(K) \leq V / \lambda$, where $V$ is the total variation of $f$ over $K$.

This argument does not work if $f$ is distributionally regulated, since in that case the set $\mathfrak{S}_{\lambda}$ could have limit points, as the next example shows.

EXAmple. Consider the function $f$ with support in $[0, \infty)$ with derivative

$$
f^{\prime}(x)=\sum_{n=1}^{\infty}(-1)^{n} n^{q} \delta\left(x-\frac{1}{n}\right)
$$

where $q \in \mathbb{R}$. Then $f$ is a distributionally regulated function, constant in all the intervals $(1 /(n+1), 1 / n)$ for $n \in \mathbb{N}$, and in $(-\infty, 0)$ where it vanishes. The set of points where $f$ has a non-zero jump is exactly $\mathfrak{S}=\{1 / n: n \in \mathbb{N}\}$. In particular, $0 \notin \mathfrak{S}$, since the function has the distributional point value $f(0)=0$. If $q>0$ then $\mathfrak{S}_{\lambda}=\mathfrak{S}$ for $\lambda \leq 1$, and thus 0 is an accumulation point of $\mathfrak{S}_{\lambda}$. Actually, we may replace the sequence $\left\{(-1)^{n} n^{q}\right\}_{n=1}^{\infty}$ by any distributionally small sequence $\left\{c_{n}\right\}_{n=1}^{\infty}$, that is, a sequence with 
$\sum_{n=1}^{\infty} c_{n} \delta(x-n)$ in $\mathcal{K}^{\prime}(\mathbb{R})[14$, Section 5.4], and still keep $f(0)=0$. Indeed,

$$
\begin{aligned}
\left\langle f^{\prime}(\varepsilon x), \phi(x)\right\rangle & =\left\langle\sum_{n=1}^{\infty} c_{n} \delta(\varepsilon x-1 / n), \phi(x)\right\rangle=\sum_{n=1}^{\infty} \frac{c_{n}}{\varepsilon} \phi\left(\frac{1}{\varepsilon n}\right) \\
& =\sum_{n=1}^{\infty} n c_{n} \tau(\varepsilon n)=o\left(\varepsilon^{\infty}\right) \quad \text { as } \varepsilon \rightarrow 0^{+},
\end{aligned}
$$

where $\tau(x)=(1 / x) \phi(1 / x)$ belongs to $\mathcal{K}(\mathbb{R})$ if $\phi \in \mathcal{D}(\mathbb{R})$, and where all series are considered in the Cesàro sense. Hence $f$ is "distributionally smooth" at $x=0$ since it follows that $f^{(m)}(0)=0$ for all $m \geq 0$.

We have the following result on the number of singularities of a distributionally regulated function.

THEOREM 8. Let $f \in \mathcal{D}^{\prime}(\mathbb{R})$ be distributionally regulated, with or without delta functions. Let

$$
\mathfrak{S}=\{x \in \mathbb{R}: f(x) \text { does not exist distributionally }\} .
$$

Then $\mathfrak{S}$ is countable at the most.

Proof. Consider first the set $\mathfrak{S}_{0}$ of those elements of $\mathfrak{S}$ where $f$ does not have delta functions. If $x_{0} \in \mathfrak{S}_{0}$ then $f\left(x_{0}^{+}\right) \neq f\left(x_{0}^{-}\right)$. Let $\phi \in \mathcal{D}(\mathbb{R})$ satisfy (4.1), and let $F(x, y)$ be the $\phi$-representation of $f$. There exists $\theta \in$ $(0, \pi / 2)$ such that

$$
\lim _{x \rightarrow x_{0}^{ \pm}} F\left(x,\left|x-x_{0}\right| \tan \theta\right)=f\left(x_{0}^{ \pm}\right), \quad \forall x_{0} \in \mathbb{R} .
$$

Let $\mathrm{U}_{0}=\{(r, \infty): r \in \mathbb{Q}\} \cup\{(-\infty, r): r \in \mathbb{Q}\}$ and let $\mathrm{U}=\left\{\left(I_{+}, I_{-}\right) \in\right.$ $\left.\mathrm{U}_{0} \times \mathrm{U}_{0}: I_{+} \cap I_{-}=\emptyset\right\}$. If $x_{0} \in \mathfrak{S}_{0}$ then there exist $\left(I_{+}, I_{-}\right) \in \mathrm{U}$ and $n \in \mathbb{N}$ such that

$$
\begin{array}{ll}
F\left(x,\left(x-x_{0}\right) \tan \theta\right) \in I_{+} & \text {for } x_{0}<x<x_{0}+1 / n, \\
F\left(x,\left(x_{0}-x\right) \tan \theta\right) \in I_{-} & \text {for } x_{0}-1 / n<x<x_{0} .
\end{array}
$$

For fixed $\left(I_{+}, I_{-}\right) \in \mathrm{U}$ and fixed $n \in \mathbb{N}$ the family of intervals $\left(x_{0}-1 / n\right.$, $\left.x_{0}+1 / n\right)$, where $x_{0} \in \mathfrak{S}_{0}$ satisfies (6.4) and (6.5), is pairwise disjoint, and consequently, there are an at most countable number of such intervals. Hence

$$
\mathfrak{S}_{0}=\bigcup_{\left(I_{+}, I_{-}\right) \in \cup} \bigcup_{n=1}^{\infty}\left\{x_{0} \in \mathbb{R}: x_{0} \text { satisfies (6.4) and (6.5) }\right\}
$$

is also countable at the most.

The analysis at points where $f$ has delta functions of a given order follows by integrating $f$ a suitable number of times. Indeed, let $\mathfrak{S}_{N}$ be the set of points of $\mathfrak{S}$ where $f$ has no delta function of order greater than $N$. Let $F$ be a primitive of $f$ of order $N+1$, i.e., $F^{(N+1)}(x)=f(x)$. Then $F$ is also a distributionally regulated function, and $\mathfrak{S}_{N} \backslash \mathfrak{S}_{N-1}$ is exactly the set of 
points where $F$ has a jump but no delta functions; hence $\mathfrak{S}_{N} \backslash \mathfrak{S}_{N-1}$ is countable at the most, and thus so is $\mathfrak{S}_{N}$. It follows that $\mathfrak{S}$ is countable at the most.

\section{Boundary behavior of solutions of partial differential equa-} tions. The results of the previous sections apply to general distributions and test functions. When the test function $\phi$ is of certain special forms, however, the $\phi$-transform becomes a particular solution of a partial differential equation, and those results become results on the boundary behavior of solutions of partial differential equations.

Suppose first that $\phi=\phi_{1}$ where

$$
\phi_{1}(x)=\frac{p(x)}{q(x)},
$$

$p$ and $q$ are polynomials, $\alpha=\operatorname{deg} q-\operatorname{deg} p \geq 2, q$ does not have real zeros, and $\int_{-\infty}^{\infty} \phi_{1}(x) d x=1$. Let

$$
q(x)=\sum_{k=0}^{n} a_{k} x^{k} .
$$

Then if $f \in \mathcal{D}^{\prime}(\mathbb{R})$ satisfies the estimate $f(x)=O\left(|x|^{\beta}\right)(\mathrm{C})$ as $|x| \rightarrow \infty$, where $\alpha+\beta<-1$, then the $\phi$-transform

$$
F_{1}(x, y)=\left\langle f(x+y \xi), \phi_{1}(\xi)\right\rangle, \quad x \in \mathbb{R}, y>0,
$$

is a solution of the partial differential equation

$$
\sum_{k=0}^{n} a_{n-k} \frac{\partial^{n} F}{\partial x^{k} \partial y^{n-k}}=0,
$$

with $F\left(x, 0^{+}\right)=f(x)$ distributionally, since

$$
\begin{aligned}
\sum_{k=0}^{n} a_{n-k} \frac{\partial^{n} F}{\partial x^{k} \partial y^{n-k}} & =\sum_{k=0}^{n} a_{n-k}\left\langle f^{(n)}(x+y \xi) \xi^{n-k}, \phi_{1}(\xi)\right\rangle \\
& =\left\langle f^{(n)}(x+y \xi) q(\xi), \phi_{1}(\xi)\right\rangle \\
& =\left\langle f^{(n)}(x+y \xi), p(\xi)\right\rangle=0 .
\end{aligned}
$$

In the particular case when $q(x)=x^{2}+1, p(x)=1 / \pi$, we obtain

$$
\phi_{2}(x)=\frac{1}{\pi\left(x^{2}+1\right)},
$$

and $F_{2}(x, y)$ is the Poisson "integral" of $f$, which in case $f(x)=O\left(|x|^{\beta}\right)(\mathrm{C})$ as $|x| \rightarrow \infty$, for some $\beta<1$, is the harmonic function with $F_{2}\left(x, 0^{+}\right)=f(x)$ distributionally that satisfies $F_{2}(x, y)=O\left(|x|^{\beta}\right)(\mathrm{C})$ as $|x| \rightarrow \infty$, for each 
fixed $y>0$. Observe that

$$
F_{2}(x, y)=\frac{y}{\pi} \int_{-\infty}^{\infty} \frac{f(\xi) d \xi}{(x-\xi)^{2}+y^{2}}
$$

if $f$ is locally integrable.

Let us now take $\phi=\varphi_{\nu}$ with Fourier transform given by

$$
\widehat{\varphi}_{\nu}(u)=e^{-u^{\nu}},
$$

where $\nu=2 p$ is an even positive integer. Alternatively, $\varphi_{\nu}$ is the only solution in $\mathcal{S}$ of the ordinary differential equation

$$
\varphi^{(\nu-1)}(\xi)=(-1)^{p} \frac{\xi}{\nu} \varphi(\xi)
$$

with $\int_{-\infty}^{\infty} \varphi(\xi) d \xi=1$. Then if $f \in \mathcal{S}^{\prime}(\mathbb{R})$, and $F$ is the $\phi$-transform corresponding to $\varphi_{\nu}$, the function

$$
G_{\nu}(x, t)=F\left(x, t^{1 / \nu}\right), \quad x \in \mathbb{R}, t>0,
$$

is a solution of the initial value problem

$$
\begin{aligned}
\frac{\partial G}{\partial t} & =(-1)^{p-1} \frac{\partial^{\nu} G}{\partial x^{\nu}} \\
G\left(x, 0^{+}\right) & =f(x) \quad \text { distributionally. }
\end{aligned}
$$

In particular, if $\nu=2$, then

$$
\widehat{\varphi}_{\nu}(u)=e^{-u^{2}}, \quad \varphi_{\nu}(\xi)=\frac{1}{2 \sqrt{\pi}} e^{-\xi^{2} / 4},
$$

and $G_{2}(x, t)$ is the solution of the heat equation $G_{, t}=G_{, x x}$ that satisfies $G\left(x, 0^{+}\right)=f(x)$ distributionally, and with $G(x, t) \in \mathcal{S}^{\prime}(\mathbb{R})$ for each fixed $t>0$. If $f$ is a locally integrable function then $G_{2}(x, t)$ takes the familiar form

$$
G_{2}(x, t)=\frac{1}{2 \sqrt{\pi t}} \int_{-\infty}^{\infty} f(\xi) e^{-(\xi-x)^{2} / 4 t} d \xi
$$

If the distributional value $f\left(x_{0}\right)=\gamma$ exists, then $F_{1}(x, y)$, and in particular $F_{2}(x, y)$, satisfies $F_{1}(x, y) \rightarrow \gamma$ as $(x, y) \rightarrow\left(x_{0}, 0\right)$ in any sector $y \geq m\left|x-x_{0}\right|$ for $m>0$. Also $G_{\nu}(x, t) \rightarrow \gamma$ in any region of the type $t \geq m\left(x-x_{0}\right)^{\nu}$ for $m>0$. Actually, if $\chi_{x_{0}}(s)=\left(f\left(x_{0}+s\right)+f\left(x_{0}-s\right)\right) / 2$, and the distributional value $\chi_{x_{0}}(0)=\gamma$ exists, then $F_{1}\left(x_{0}, y\right) \rightarrow \gamma$ as $y \rightarrow 0^{+}$ and $G_{\nu}\left(x_{0}, t\right) \rightarrow \gamma$ as $t \rightarrow 0^{+}$. If instead of the existence of the distributional value one just has the existence of the distributional limit $f\left(x_{0}^{ \pm}\right)=\gamma$, then the finite part of the limit of $F_{1}(x, y)$ as $(x, y) \rightarrow\left(x_{0}, 0\right)$ in any sector $y \geq m\left|x-x_{0}\right|$ exists and equals $\gamma$; similarly, one obtains the existence of the finite part of the limits in the other cases. 
REMARK. In principle one can take a test function in (7.1) with $\operatorname{deg} q-$ $\operatorname{deg} p=1$. For example, we can take $\phi(x)=(2 \pi i)^{-1}(x \mp i)^{-1}$, but the results will not hold, since in this case the $\phi$-transform becomes the analytic representation, which obeys different rules [13, Chapter 6]; actually even Theorem 1 does not hold for the analytic representation.

It is interesting to observe that if $f$ is almost periodic or periodic, then

$$
f(x)=\sum_{n=-\infty}^{\infty} a_{n} e^{i \alpha_{n} x},
$$

where $\alpha_{n} \rightarrow \pm \infty$ as $n \rightarrow \pm \infty$. It follows that

$$
F(x, y)=\sum_{n=-\infty}^{\infty} a_{n} e^{i \alpha_{n} x} \widehat{\phi}\left(\alpha_{n} y\right)
$$

so that in particular

$$
F_{2}(x, y)=\sum_{n=-\infty}^{\infty} a_{n} e^{i \alpha_{n} x} e^{-\left|\alpha_{n}\right| y}=\sum_{n=-\infty}^{\infty} a_{n} e^{i \alpha_{n} x} r^{\left|\alpha_{n}\right|},
$$

where $r=e^{-y} \rightarrow 1^{-}$as $y \rightarrow 0^{+}$. The study of the behavior of the $\phi$-transform in this case becomes the study of the series (7.13) in the Abel sense. Also

$$
G_{\nu}(x, t)=\sum_{n=-\infty}^{\infty} a_{n} e^{i \alpha_{n} x} e^{-\left|\alpha_{n}\right|^{\nu} t}
$$

The problem of finding the (ordinary) jumps of a Fourier series was first solved by Fejér [15] in terms of the partial sums of the series, and was later considered by Zygmund [39, 9.11, Chapter III, §108] in terms of the AbelPoisson means of the Fourier series. A different formula using logarithmic means was given by Lukács [22], [39, Thm. 8.13], and recently studied in terms of the Abel-Poisson means by Móricz [25] for point values of the first order and for general distributional point values in [11]. Theorems 6 and 7 provide very general results of the Fejér and Lukács type, respectively, for a general test function $\phi$ (which provides many different types of summability means, such as (7.15) or (7.16)) and not only for Fourier series, but also for non-harmonic series and actually for any distribution.

8. The Fourier transform of regulated functions. In this section we shall characterize the Fourier transform of distributionally regulated functions, with or without delta functions. We first start with some comments on distributional evaluations and the notation used for them.

Let $f \in \mathcal{D}^{\prime}(\mathbb{R})$ with support bounded on the left. If $\phi \in \mathcal{E}(\mathbb{R})$ then the evaluation $\langle f(x), \phi(x)\rangle$ will not be defined, in general. We say that the 
evaluation exists in the Cesàro sense and equals $L$, written as

$$
\langle f(x), \phi(x)\rangle=L \quad(\mathrm{C})
$$

if $g(x)=L+o(1)(\mathrm{C})$ as $x \rightarrow \infty$, where $g$ is the primitive of $f \phi$ with support bounded on the left. A similar definition applies if $\operatorname{supp} f$ is bounded on the right. Observe that if $f$ is locally integrable with supp $f \subset[a, \infty)$ then (8.1) means that

$$
\int_{a}^{\infty} f(x) \phi(x) d x=L \quad(\mathrm{C}),
$$

while if $f(x)=\sum_{n=0}^{\infty} a_{n} \delta(x-n)$ then (8.1) tells us that

$$
\sum_{n=0}^{\infty} a_{n} \phi(n)=L \quad(\mathrm{C}) .
$$

In the general case when the support of $f$ extends to both $-\infty$ and $+\infty$, there are various different but related notions of evaluations in the Cesàro sense (or in any other summability sense, in fact). If $f$ admits a representation of the form $f=f_{1}+f_{2}$, with supp $f_{1}$ bounded on the left and supp $f_{2}$ bounded on the right, such that $\left\langle f_{j}(x), \phi(x)\right\rangle=L_{j}(\mathrm{C})$ exist, then we say that the $(\mathrm{C})$ evaluation $\langle f(x), \phi(x)\rangle(\mathrm{C})$ exists and equals $L=L_{1}+L_{2}$. This is clearly independent of the decomposition. The notation (8.1) is used in this situation.

It often happens that $\langle f(x), \phi(x)\rangle(\mathrm{C})$ does not exist, but the symmetric limit, $\lim _{x \rightarrow \infty}\{g(x)-g(-x)\}=L$, where $g$ is any primitive of $f \phi$, exists in the $(\mathrm{C})$ sense. Then we say that the evaluation $\langle f(x), \phi(x)\rangle$ exists in the principal value Cesàro sense, and write

$$
\text { p.v. }\langle f(x), \phi(x)\rangle=L \quad(\mathrm{C}) .
$$

Observe that p.v. $\sum_{n=-\infty}^{\infty} a_{n} \phi(n)=L(\mathrm{C})$ if and only if $\sum_{n=-N}^{N} a_{n} \phi(n)$ $\rightarrow L(\mathrm{C})$ as $N \rightarrow \infty$, while p.v. $\int_{-\infty}^{\infty} f(x) \phi(x) d x=L(\mathrm{C})$ if and only if $\int_{-A}^{A} f(x) \phi(x) d x \rightarrow L(\mathrm{C})$ as $A \rightarrow \infty$.

An intermediate notion, very useful for our purposes, is the following. If there exists $k$ such that

$$
\lim _{x \rightarrow \infty}\{g(a x)-g(-x)\}=L \quad(\mathrm{C}, k), \forall a>0,
$$

we say that the distributional evaluation exists in the e.v. Cesàro sense and write

$$
\text { e.v. }\langle f(x), \phi(x)\rangle=L \quad(\mathrm{C}, k),
$$

or just e.v. $\langle f(x), \phi(x)\rangle=L(\mathrm{C})$ if there is no need to call attention to the value of $k$; observe, however, that the same value of $k$ works for all $a>0$.

Clearly $(8.1) \Rightarrow(8.6) \Rightarrow(8.4)$, but the converse implications do not hold. For example, p.v. $\langle x, 1\rangle=0$ (no $(\mathrm{C})$ needed), but e.v. $\langle x, 1\rangle(\mathrm{C})$ does not 
exist. Furthermore,

$$
\text { e.v. }\left\langle\sum_{\substack{n=-\infty \\|n| \geq 2}}^{\infty} \frac{\delta(x-n)}{n \ln |n|}, 1\right\rangle=0
$$

with no $(\mathrm{C})$ needed, but the Cesàro evaluation does not exist in the sense of (8.1).

Our next aim is to characterize the Fourier transforms of distributions that have a jump discontinuity at a point. The characterization of the Fourier series of those periodic distributions that have a distributional point value was given in [8]: if $f(\theta)=\sum_{n=-\infty}^{\infty} a_{n} e^{i n \theta}$ in the space $\mathcal{D}^{\prime}(\mathbb{R})$ then

$$
f\left(\theta_{0}\right)=\gamma \quad \text { distributionally }
$$

if and only if there exists $k$ such that

$$
\lim _{x \rightarrow \infty} \sum_{-x \leq n \leq a x} a_{n} e^{i n \theta_{0}}=\gamma \quad(\mathrm{C}, k), \forall a>0 .
$$

We shall show that a similar result holds for Fourier transforms.

LEMмA 1. Let $f \in \mathcal{S}^{\prime}(\mathbb{R})$. If $x_{0} \in \mathbb{R}$ then

$$
f\left(x_{0}\right)=\gamma \quad \text { distributionally }
$$

if and only if

$$
\lim _{\lambda \rightarrow \infty} \lambda \widehat{f}(\lambda u) e^{-i \lambda u x_{0}}=2 \pi \gamma \delta(u)
$$

in the space $\mathcal{S}^{\prime}(\mathbb{R})$.

Proof. Indeed,

$$
\begin{aligned}
f\left(x_{0}\right)=\gamma \text { dist. } & \Leftrightarrow \lim _{\varepsilon \rightarrow 0} f\left(x_{0}+\varepsilon x\right)=\gamma \\
& \Leftrightarrow \lim _{\varepsilon \rightarrow 0} \mathcal{F}\left\{f\left(x_{0}+\varepsilon x\right) ; u\right\}=2 \pi \gamma \delta(u) \\
& \Leftrightarrow \lim _{\lambda \rightarrow \infty} \lambda \widehat{f}(\lambda u) e^{-i \lambda u x_{0}}=2 \pi \gamma \delta(u),
\end{aligned}
$$

as required.

In what follows we use the notation spec $f=\operatorname{supp} \widehat{f}$ for the spectrum of $f$. The next lemma follows from the ideas of [14, Section 6.5]; see also [36].

Lemma 2. Let $f \in \mathcal{S}^{\prime}(\mathbb{R})$. Suppose spec $f$ is bounded on the left or on the right. Then $f\left(x_{0}\right)=\gamma$ distributionally if and only if

$$
\left\langle\widehat{f}(u), e^{-i u x_{0}}\right\rangle=2 \pi \gamma \quad(\mathrm{C}) .
$$

Our next lemma concerns the case of a distribution that vanishes on a whole interval. 
Lemma 3. Let $f \in \mathcal{S}^{\prime}(\mathbb{R})$. Suppose $f(x)=0$ for $x_{0}-\eta<x<x_{0}+\eta$. Then $f$ admits a decomposition $f=f_{+}-f_{-}$with $f_{ \pm} \in \mathcal{S}^{\prime}(\mathbb{R})$, where $\operatorname{spec} f_{+}$ is bounded on the right, spec $f_{-}$is bounded on the left, and where the distributional point values $f_{ \pm}\left(x_{0}\right)= \pm \mu$ both exist.

Proof. If $f \in \mathcal{D}^{\prime}(\mathbb{R})$ then ([4, Theorem 3.14], [26]) there exists a sectionally analytic function $F(z)$ defined for $z \in \mathbb{C} \backslash \mathbb{R}$ such that the distributional limits

$$
f_{ \pm}(x)=F(x \pm i 0)=\lim _{y \rightarrow 0} F(x \pm i y)
$$

exist and $f=f_{+}-f_{-}$. When $f \in \mathcal{S}^{\prime}(\mathbb{R})$ we can choose the function $F$ in such a way that both $f_{ \pm} \in \mathcal{S}^{\prime}(\mathbb{R})\left[13\right.$, Section 6.4]; actually if $f(x)=O\left(|x|^{\beta}\right)(\mathrm{C})$ as $x \rightarrow \infty$ for some $\beta<0$, then we may take

$$
F(z)=\frac{1}{2 \pi i}\left\langle f(x), \frac{1}{x-z}\right\rangle
$$

In general $F$ is not unique, but an arbitrary polynomial can be added at will.

It is clear that $\operatorname{spec} f_{+} \subset(-\infty, 0]$ while spec $f_{-} \subset[0, \infty)$.

It remains to show that the distributional point values $f_{ \pm}\left(x_{0}\right)$ exist. But since $f(x)=0$ for $x_{0}-\eta<x<x_{0}+\eta$ it follows that $F$ is analytic across this interval [2, Section 5.8], and thus $f_{ \pm}(x)$ are actually real analytic functions for $x_{0}-\eta<x<x_{0}+\eta$ and thus $f_{ \pm}\left(x_{0}\right)$ are well-defined ordinary values.

We are now ready to give the characterization of the Fourier transforms of tempered distributions that have a distributional point value.

Theorem 9. Let $f \in \mathcal{S}^{\prime}(\mathbb{R})$. If $x_{0} \in \mathbb{R}$ then

$$
f\left(x_{0}\right)=\gamma \quad \text { distributionally }
$$

if and only if

$$
\text { e.v. }\left\langle\widehat{f}(u), e^{-i u x_{0}}\right\rangle=2 \pi \gamma \quad(\mathrm{C}),
$$

which in case $\widehat{f}$ is locally integrable means that

$$
\text { e.v. } \int_{-\infty}^{\infty} \widehat{f}(u) e^{-i u x_{0}} d u=2 \pi \gamma
$$

Proof. Choose any number $\eta$ with $0<\eta<\pi$. There exists a distribution $f_{1}$ of period $2 \pi$ such that $f(x)=f_{1}(x)$ for $x_{0}-\eta<x<x_{0}+\eta$. This means, because of Lemma 3 , that $f=f_{1}+f_{2}$ where $f_{2}$ satisfies e.v. $\left\langle\widehat{f}_{2}(u), e^{-i u x_{0}}\right\rangle=0$ $(\mathrm{C})$, and thus the result will be true if it is true for periodic distributions of period $2 \pi$, but this is exactly the equivalence of (8.8) and (8.9) proved in $[8]$. 
We now proceed to the case of tempered distributions that have distributional lateral limits at a point.

TheOREM 10. Let $f \in \mathcal{S}^{\prime}(\mathbb{R})$. If $x_{0} \in \mathbb{R}$ then the distributional lateral limits $f\left(x_{0}^{ \pm}\right)=\gamma_{ \pm}$exist and $f$ has no Dirac delta function at $x=x_{0}$ if and only if there exists $k$ such that whenever $g(u)$ is a primitive of $\widehat{f}(u) e^{-i u x_{0}}$ then the Cesàro limit

$$
\lim _{u \rightarrow \infty}(g(a u)-g(-u))=I_{x_{0}}(a) \quad(\mathrm{C}, k)
$$

exists for all $a>0$. If this is the case then

$$
I_{x_{0}}(a)=\pi\left(\gamma_{+}+\gamma_{-}\right)+i\left(\gamma_{+}-\gamma_{-}\right) \ln a .
$$

Proof. Suppose that the distributional lateral limits $f\left(x_{0}^{ \pm}\right)=\gamma_{ \pm}$exist and $f$ has no Dirac delta function at $x=x_{0}$. Write $f=f_{1}+f_{2}$ where $f_{1}(x)=f(x)-(d / 2) \operatorname{sgn}\left(x-x_{0}\right), d=\gamma_{+}-\gamma_{-}$. Thus for $f=f_{1}$, the quantity $I_{x_{0}, f_{1}}(a)$ exists and equals $\pi\left(\gamma_{+}+\gamma_{-}\right)$since the distributional point value $f_{1}\left(x_{0}\right)$ exists and equals $\left(\gamma_{+}+\gamma_{-}\right) / 2$, and therefore

$$
\text { e.v. }\left\langle\widehat{f}_{1}(u), e^{-i u x_{0}}\right\rangle=\pi\left(\gamma_{+}+\gamma_{-}\right)
$$

On the other hand,

$$
\widehat{f}_{2}(u)=\left(\gamma_{+}-\gamma_{-}\right) i e^{i u x_{0}} \text { p.v. }\left(\frac{1}{u}\right),
$$

where p.v. $(1 / u)$ is the usual principal value regularization of the non-integrable function $1 / u$. Then

$$
\begin{aligned}
I_{x_{0}, f_{2}}(a) & =\lim _{s \rightarrow \infty} \text { p.v. } \int_{-s}^{a s} \widehat{f}_{2}(u) e^{-i u x_{0}} d u \\
& =\lim _{s \rightarrow \infty} \text { p.v. } \int_{-s}^{a s}\left(\gamma_{+}-\gamma_{-}\right) i \frac{d u}{u}=\left(\gamma_{+}-\gamma_{-}\right) i \ln a,
\end{aligned}
$$

and (8.18) and (8.19) follow.

Conversely, suppose that $I_{x_{0}}(a)$ exists for each $a>0$. Clearly $I_{x_{0}}(a)$ is a measurable function of $a$. Then an easy computation shows that $I_{x_{0}}(a)$ satisfies the functional equation

$$
I_{x_{0}}(a b)=I_{x_{0}}(a)+I_{x_{0}}(b)-I_{x_{0}}(1) .
$$

While this functional equation has many solutions, constructed using a suitable Hamel basis, an analysis that can be traced back to Sierpiński shows that the only measurable solutions are

$$
I_{x_{0}}(a)=I_{x_{0}}(1)+\omega \ln a
$$


for some constant $\omega$. Writing $f=f_{1}+f_{2}, f_{2}(x)=-i(\omega / 2) \operatorname{sgn}\left(x-x_{0}\right)$ shows that the distributional value $f_{1}\left(x_{0}\right)$ exists and equals $I_{x_{0}}(1) /(2 \pi)$ since

$$
\text { e.v. }\left\langle\widehat{f}_{1}(u), e^{-i u x_{0}}\right\rangle=I_{x_{0}}(1) \quad(\mathrm{C}) .
$$

Hence the distributional lateral limits $f\left(x_{0}^{ \pm}\right)$exist and equal

$$
\gamma_{ \pm}=\frac{I_{x_{0}}(1)}{2 \pi} \mp \frac{i \omega}{2}
$$

which is equivalent to $(8.19)$.

Observe in particular that if $\widehat{f}$ is locally integrable, then the distributional lateral limits $f\left(x_{0}^{ \pm}\right)=\gamma_{ \pm}$exist and $f$ has no Dirac delta function at $x=x_{0}$ if and only if there exists $k$ such that for all $a>0$,

$$
\lim _{s \rightarrow \infty} \int_{-s}^{a s} \widehat{f}(u) e^{-i u x_{0}} d u=\pi\left(\gamma_{+}+\gamma_{-}\right)+i\left(\gamma_{+}-\gamma_{-}\right) \ln a \quad(\mathrm{C}, k) .
$$

In case $f$ is periodic of period $2 \pi$ with Fourier series

$$
f(x)=\sum_{n=-\infty}^{\infty} a_{n} e^{i n x}
$$

the condition becomes

$$
\text { (8.28) } \lim _{N \rightarrow \infty} \sum_{-a N \leq n \leq N} a_{n} e^{i n x_{0}}=\frac{\gamma_{+}+\gamma_{-}}{2}+\frac{i}{2 \pi}\left(\gamma_{+}-\gamma_{-}\right) \ln a \quad(\mathrm{C}, k) \text {. }
$$

We obtain the following characterization of the Fourier transforms of distributionally regulated functions.

THEOREM 11. Let $f \in \mathcal{S}^{\prime}(\mathbb{R})$. The distribution $f$ is a distributionally regulated function with delta functions if and only if for all $x_{0} \in \mathbb{R}$, the distribution $\widehat{f}(u) e^{-i u x_{0}}$ admits the decomposition

$$
\widehat{f}(u) e^{-i u x_{0}}=p_{x_{0}}(u)+g_{x_{0}}^{\prime}(u),
$$

where $p_{x_{0}}(u)$ is a polynomial and where for some $k$,

$$
\lim _{u \rightarrow \infty}(g(a u)-g(-u))=I_{x_{0}}(a) \quad(\mathrm{C}, k)
$$

exists for all $a>0$. The distribution $f$ is a distributionally regulated function (without delta functions) if $p_{x_{0}}(u)=0$ for each $x_{0} \in \mathbb{R}$; if also $I_{x_{0}}(a)$ is a constant function of a for each $x_{0} \in \mathbb{R}$ then $f$ is a Eojasiewicz function.

In any case, the set of points $x_{0}$ where $p_{x_{0}}(u) \neq 0$ is countable, as is the set of points $x_{0}$ where $I_{x_{0}}(a)$ is not a constant function of $a$.

We now give another characterization of distributions having lateral limits based on a decomposition in terms of boundary limits of analytic functions from the upper and lower half planes. Observe that only principal value Cesàro evaluations are needed in the following theorem. 
Theorem 12. Let $f \in \mathcal{S}^{\prime}(\mathbb{R})$. Let $x_{0} \in \mathbb{R}$. Then the distributional lateral limits $f\left(x_{0}^{ \pm}\right)=\gamma_{ \pm}$exist and $f$ has no Dirac delta function at $x=x_{0}$ if and only if

$$
\widehat{f}(u) e^{-i u x_{0}}=H_{x_{0}}(u+i 0)+H_{x_{0}}(u-i 0),
$$

where $H_{x_{0}}(z)$ is analytic for $z \in \mathbb{C} \backslash \mathbb{R}$, the distributional boundary distributions $H_{x_{0}}(u \pm i 0)$ belong to $\mathcal{S}^{\prime}(\mathbb{R})$, and the principal value Cesàro evaluations

$$
\text { p.v. }\left\langle H_{x_{0}}(u \pm i 0), 1\right\rangle=\nu_{ \pm}
$$

both exist. In this case $\nu_{ \pm}=\pi \gamma_{ \pm}$.

Proof. If the distributional lateral limits $f\left(x_{0}^{ \pm}\right)=\gamma_{ \pm}$exist and $f$ has no Dirac delta function at $x=x_{0}$ we can write $f=f_{+}+f_{-}$where $f_{ \pm}$do not have delta functions at $x=x_{0}, \operatorname{supp} f_{+} \subset\left[x_{0}, \infty\right), \operatorname{supp} f_{-} \subset\left(-\infty, x_{0}\right]$, $f_{+}\left(x_{0}^{+}\right)=\gamma_{+}$, and $f_{-}\left(x_{0}^{-}\right)=\gamma_{-}$. Then we define

$$
H_{x_{0}}(z)= \begin{cases}\left\langle f_{+}(x), e^{i z\left(x-x_{0}\right)}\right\rangle, & \operatorname{Re} z>0, \\ \left\langle f_{-}(x), e^{i z\left(x-x_{0}\right)}\right\rangle, & \operatorname{Re} z<0,\end{cases}
$$

so that $H_{x_{0}}(u \pm i 0)=\widehat{f}_{ \pm}(u)$, and consequently

$$
\text { p.v. }\left\langle H_{x_{0}}(u \pm i 0), 1\right\rangle=\pi \gamma_{ \pm} \quad(\mathrm{C}) .
$$

Conversely, if (8.31) holds, then $f=f_{+}+f_{-}$where

$$
f_{ \pm}(x)=\mathcal{F}^{-1}\left\{e^{i u x_{0}} H_{x_{0}}(u \pm i 0), x\right\} .
$$

But this implies that $\operatorname{supp} f_{+} \subset\left[x_{0}, \infty\right)$, while supp $f_{-} \subset\left(-\infty, x_{0}\right]$. Then (8.32) shows that the even parts of $f_{ \pm}$have the distributional values $\gamma_{ \pm} / 2$ at $x=x_{0}$. But since the distributions $f_{ \pm}$vanish on one side of $x_{0}$, it follows that the distributional lateral limits exist and no delta function is present.

We immediately obtain the ensuing result.

TheOREM 13. Let $f \in \mathcal{S}^{\prime}(\mathbb{R})$. The distribution $f$ is a distributionally regulated function with delta functions if and only if for all $x_{0} \in \mathbb{R}$, the distribution $\widehat{f}(u) e^{-i u x_{0}}$ admits the decomposition

$$
\widehat{f}(u) e^{-i u x_{0}}=p_{x_{0}}(u)+H_{x_{0}}(u+i 0)+H_{x_{0}}(u-i 0),
$$

where $p_{x_{0}}(u)$ is a polynomial and where $H_{x_{0}}(z)$ is analytic for $z \in \mathbb{C} \backslash \mathbb{R}$, the distributional boundary distributions $H_{x_{0}}(u \pm i 0)$ belong to $\mathcal{S}^{\prime}(\mathbb{R})$, and the principal value Cesàro evaluations

$$
\text { p.v. }\left\langle H_{x_{0}}(u \pm i 0), 1\right\rangle=\nu_{ \pm}
$$

both exist. The distribution $f$ is a distributionally regulated function (without delta functions) if $p_{x_{0}}(u)=0$ for each $x_{0} \in \mathbb{R}$; if also $\nu_{+}=\nu_{-}$for each $x_{0} \in \mathbb{R}$ then $f$ is a Lojasiewicz function. 
In any case the set of points $x_{0} \in \mathbb{R}$ where $p_{x_{0}}(u) \neq 0$ is countable, as is the set of points where $\nu_{+} \neq \nu_{-}$.

One can use these ideas to prove that if the distributional lateral limits of a distribution that is the boundary value of an analytic function from the upper or lower half plane exist, then they must coincide [10].

\section{References}

[1] R. G. Bartle, A Modern Theory of Integration, Amer. Math. Soc., Providence, 2001.

[2] E. J. Beltrami and M. R. Wohlers, Distributions and the Boundary Values of Analytic Functions, Academic Press, New York, 1966.

[3] J. Boersma, A nonharmonic trigonometric series, SIAM Rev. 37 (1995), 443-445.

[4] H. Bremermann, Distributions, Complex Variables and Fourier Transforms, Addison-Wesley, Reading, MA, 1965.

[5] J. Campos Ferreira, Introduction to the Theory of Distributions, Longman, Essex, 1997.

[6] J. Dieudonné, Foundations of Modern Analysis, Academic Press, New York, 1969.

[7] A. L. Durán, R. Estrada and R. P. Kanwal, Extensions of the Poisson summation formula, J. Math. Anal. Appl. 218 (1998), 581-606.

[8] R. Estrada, Characterization of the Fourier series of distributions having a value at a point, Proc. Amer. Math. Soc. 124 (1996), 1205-1212.

[9] - The Cesàro behaviour of distributions, Proc. Roy. Soc. London Ser. A 454 (1998), $2425-2443$.

[10] -, Abel summability and angular convergence, Scientia 9 (2003), 45-51.

[11] - A distributional version of the Ferenc Lukács theorem, Sarajevo J. Math. 1 (2005), 1-17.

[12] -, Distributional radius of curvature, Math. Methods Appl. Sci. 29 (2006), 427-444.

[13] R. Estrada and R. P. Kanwal, Singular Integral Equations, Birkhäuser, Boston, 2000.

[14] —, 一, A Distributional Approach to Asymptotics. Theory and Applications, 2nd ed., Birkhäuser, Boston, 2002.

[15] L. Fejér, Über die Bestimmung des Sprunges der Funktion aus ihrer Fourierreihe, J. Reine Angew. Math. 142 (1913), 165-188. See also the collected works of L. Fejér: Gesammelte Arbeiten, Akadémiai Kiadó, Budapest, 1970, 718-744.

[16] M. L. Glasser, A nonharmonic trigonometric series, SIAM Rev. 37 (1995), 443.

[17] R. A. Gordon, The Integrals of Lebesgue, Denjoy, Perron, and Henstock, Amer. Math. Soc., Providence, 1994.

[18] A. Grossman, G. Loupias and E. M. Stein, An algebra of pseudodifferential operators and quantum mechanics in phase space, Ann. Inst. Fourier (Grenoble) 18 (1968), 343-368.

[19] J. Horváth, Topological Vector Spaces and Distributions, Vol. I, Addison-Wesley, Reading, MA, 1966.

[20] R. P. Kanwal, Generalized Functions: Theory and Technique, 2nd ed., Birkhäuser, Boston, 1998.

[21] S. Łojasiewicz, Sur la valeur et la limite d'une distribution en un point, Studia Math. 16 (1957), 1-36. 
[22] F. Lukács, Über die Bestimmung des Sprunges einer Funktion aus ihrer Fourierreihe, J. Reine Angew. Math. 150 (1920), 107-112.

[23] J. Lützen, The Prehistory of the Theory of Distributions, Springer, New York, 1982.

[24] O. P. Misra and J. Lavoine, Transform Analysis of Generalized Functions, NorthHolland, Amsterdam, 1986.

[25] F. Móricz, Ferenc Lukács type theorems in terms of the Abel-Poisson mean of conjugate series, Proc. Amer. Math. Soc. 131 (2003), 1243-1250.

[26] M. Orton, Hilbert transforms, Plemelj relations, and Fourier transforms, SIAM J. Math. Anal. 4 (1973), 656-670.

[27] J. Peetre, On the value of a distribution at a point, Portugal. Math. 27 (1968), 149-159.

[28] S. Pilipović, B. Stanković and A. Takači, Asymptotic Behaviour and Stieltjes Transformation of Distributions, Teubner-Texte Math. 116, Teubner, Leipzig, 1990.

[29] Ch. Pommerenke, Boundary Behaviour of Conformal Maps, Springer, Berlin, 1992.

[30] L. Schwartz, Théorie des Distributions, Hermann, Paris, 1966.

[31] V. S. Vladimirov, Yu. N. Drozhzhinov and B. I. Zav'yalov, Multidimensional Tauberian Theorems for Generalized Functions, Nauka, Moscow, 1986 (in Russian).

[32] G. Walter, Pointwise convergence of distributional expansions, Studia Math. 26 (1966), 143-154.

[33] —, Local boundary behavior of harmonic functions, Illinois J. Math. 16 (1972), 491-501.

[34] -, Sampling bandlimited functions of polynomial growth, SIAM J. Math. Anal. 19 (1988), 1198-1203.

[35] - Abel summability for a distribution sampling theorem, in: Generalized Functions, Convergence Structures, and Their Applications, B. Stanković et al. (eds.), Plenum Press, New York, 1988, 349-357.

[36] -, On the value of a distribution at a point, ibid., 359-364.

[37] T. Xin and H. Wong, A delta function model of facets, Surface Sci. Lett. 487 (2002), L529-L533.

[38] A. H. Zemanian, Generalized Integral Transforms, Interscience, New York, 1965.

[39] A. Zygmund, Trigonometric Series, 3rd ed., Cambridge Univ. Press, Cambridge, 2002 .

Department of Mathematics

Louisiana State University

Baton Rouge, LA 70803-4918, U.S.A.

E-mail: jvindas@math.lsu.edu restrada@math.lsu.edu

Received January 26, 2006

Revised version May 24, 2007 